University of Wollongong

Research Online

Australian Institute for Innovative Materials -

Papers

Australian Institute for Innovative Materials

$1-1-2020$

Magnetic Modulation of Terahertz Waves via Spin-Polarized Electron

Tunneling Based on Magnetic Tunnel Junctions

Zuanming Jin

Jugeng Li

Wenjie Zhang

Chenyang Guo

Caihua Wan

See next page for additional authors

Follow this and additional works at: https://ro.uow.edu.au/aiimpapers

Part of the Engineering Commons, and the Physical Sciences and Mathematics Commons

Research Online is the open access institutional repository for the University of Wollongong. For further information contact the UOW Library: research-pubs@uow.edu.au 


\title{
Magnetic Modulation of Terahertz Waves via Spin-Polarized Electron Tunneling Based on Magnetic Tunnel Junctions
}

\author{
Abstract \\ (C) 2020 American Physical Society. Magnetic tunnel junctions (MTJs) are a key technology in modern \\ spintronics because they are the basis of read-heads of modern hard disk drives, nonvolatile magnetic \\ random access memories, and sensor applications. In this paper, we demonstrate that tunneling \\ magnetoresistance can influence terahertz $(\mathrm{THz})$ wave propagation through a MTJ. In particular, various \\ magnetic configurations between parallel state and antiparallel state of the magnetizations of the \\ ferromagnetic layers in the MTJ have the effect of changing the conductivity, making a functional \\ modulation of the propagating $\mathrm{THz}$ electromagnetic fields. Operating in the $\mathrm{THz}$ frequency range, a \\ maximal modulation depth of $60 \%$ is reached for the parallel state of the MTJ with a thickness of 77.45 \\ $\mathrm{nm}$, using a magnetic field as low as $30 \mathrm{mT}$. The THz conductivity spectrum of the MTJ is governed by \\ spin-dependent electron tunneling. It is anticipated that the MTJ device and its tunability scheme will have \\ many potential applications in $\mathrm{THz}$ magnetic modulators, filtering, and sensing.

\section{Disciplines} \\ Engineering | Physical Sciences and Mathematics

\section{Publication Details} \\ Jin, Z., Li, J., Zhang, W., Guo, C., Wan, C., Han, X., Cheng, Z., Zhang, C., Balakin, A., Shkurinov, A., Peng, Y., \\ Ma, G., Zhu, Y., Yao, J. \& Zhuang, S. (2020). Magnetic Modulation of Terahertz Waves via Spin-Polarized \\ Electron Tunneling Based on Magnetic Tunnel Junctions. Physical Review Applied, 14 (1),

\section{Authors} \\ Zuanming Jin, Jugeng Li, Wenjie Zhang, Chenyang Guo, Caihua Wan, Xiufeng Han, Zhenxiang Cheng, C \\ Zhang, Alexey Balakin, Alexander Shkurinov, Yan Peng, Guohong Ma, Yiming Zhu, Jianquan Yao, and \\ Songlin Zhuang
}




\title{
Magnetic Modulation of Terahertz Waves via Spin-Polarized Electron Tunneling Based on Magnetic Tunnel Junctions
}

\author{
Zuanming Jin $\odot,{ }^{1,2,3, *, \uparrow}$ Jugeng Li $\odot,{ }^{2, \uparrow}$ Wenjie Zhang, ${ }^{2}$ Chenyang Guo, ${ }^{4}$ Caihua Wan, ${ }^{4, \dagger}$ \\ Xiufeng Han $\odot,{ }^{4}$ Zhenxiang Cheng $\odot,{ }^{5}$ Chao Zhang, ${ }^{6}$ Alexey V. Balakin $\odot,{ }^{1,7}$ \\ Alexander P. Shkurinov, ${ }^{1,7}$ Yan Peng, ${ }^{1,3}$ Guohong Ma, ${ }^{2,8, \$}$ Yiming Zhu, ${ }^{1,3,8}$ Jianquan Yao, ${ }^{9}$ \\ and Songlin Zhuang ${ }^{1,3}$ \\ ${ }^{1}$ Terahertz Technology Innovation Research Institute, Terahertz Spectrum and Imaging Technology Cooperative \\ Innovation Center, Shanghai Key Lab of Modern Optical System, University of Shanghai for Science and \\ Technology, 516 JunGong Road, Shanghai 200093, China \\ ${ }^{2}$ Department of Physics, Shanghai University, 99 Shangda Road, Shanghai 200444, China \\ ${ }^{3}$ Shanghai Institute of Intelligent Science and Technology, Tongji University, Shanghai 200092, China \\ ${ }^{4}$ Beijing National Laboratory for Condensed Matter Physics, Institute of Physics, University of Chinese Academy \\ of Sciences, Chinese Academy of Sciences, Beijing 100190, China \\ ${ }^{5}$ Institute for Superconducting and Electronic Materials, University of Wollongong, Wollongong, New South Wales \\ 2500, Australia \\ ${ }^{6}$ School of Physics, University of Wollongong, Wollongong, New South Wales 2522, Australia \\ ${ }^{7}$ Department of Physics and International Laser Center, Lomonosov Moscow State University, Leninskie Gory 1, \\ Moscow 19991 Russia \\ ${ }^{8}$ STU \& SIOM Joint Laboratory for Superintense Lasers and Applications, Shanghai 201210, China \\ ${ }^{9}$ College of Precision Instrument and Optoelectronics Engineering, Tianjin University, Tianjin 300110, China
}

(Received 22 February 2020; revised 26 May 2020; accepted 19 June 2020; published 13 July 2020)

\begin{abstract}
Magnetic tunnel junctions (MTJs) are a key technology in modern spintronics because they are the basis of read-heads of modern hard disk drives, nonvolatile magnetic random access memories, and sensor applications. In this paper, we demonstrate that tunneling magnetoresistance can influence terahertz ( THz) wave propagation through a MTJ. In particular, various magnetic configurations between parallel state and antiparallel state of the magnetizations of the ferromagnetic layers in the MTJ have the effect of changing the conductivity, making a functional modulation of the propagating $\mathrm{THz}$ electromagnetic fields. Operating in the $\mathrm{THz}$ frequency range, a maximal modulation depth of $60 \%$ is reached for the parallel state of the MTJ with a thickness of $77.45 \mathrm{~nm}$, using a magnetic field as low as $30 \mathrm{mT}$. The $\mathrm{THz}$ conductivity spectrum of the MTJ is governed by spin-dependent electron tunneling. It is anticipated that the MTJ device and its tunability scheme will have many potential applications in $\mathrm{THz}$ magnetic modulators, filtering, and sensing.
\end{abstract}

DOI: 10.1103/PhysRevApplied.14.014032

\section{INTRODUCTION}

Terahertz $(\mathrm{THz})$ radiation lies in the frequency range from 0.1 to $10 \mathrm{THz}$, between the microwave and infrared regions $[1,2]$. Recent technological innovation in photonics and nanotechnology is enabling $\mathrm{THz}$ technology to be applied in information and communications [3,4], imaging and spectroscopy [5,6], biology and medicine [7,8], nondestructive evaluation and security [9]. Among the potential $\mathrm{THz}$ applications, imaging and communications

\footnotetext{
*physics_jzm@usst.edu.cn

†wancaihua@iphy.ac.cn

$\ddagger$ ghma@staff.shu.edu.cn

§ymzhu@usst.edu.cn

"These authors contributed equally to this work.
}

are two important research subjects that may bring great breakthroughs in modern optoelectronic information technology. Therefore, suitable functional devices and materials are in high demand for modulation or control of $\mathrm{THz}$ radiation $[10,11]$. THz modulation has been demonstrated by optical, electronic, thermal, and mechanical methods [12-16]. Usually, optical methods require an intense ultrashort femtosecond laser beam, while thermal methods are limited to the scale of tens of milliseconds. In addition, these traditional modulation methods are not entirely suitable for the $\mathrm{THz}$ wave bandwidth.

It is noteworthy that $\mathrm{THz}$ transients have become increasingly important for investigation of the light-matter interaction in spintronic materials, since many dynamic processes such as spin currents [17-19] and magnetic spin waves $[20,21]$ oscillate with $\mathrm{THz}$ frequencies. There 
have been numerous $\mathrm{THz}$ devices controlled by external magnetic fields demonstrated for amplitude modulation [22-24], phase retardation [25], and polarization control $[26,27]$. Recently, liquid-suspended magnetic ferrofluid $\mathrm{Fe}_{3} \mathrm{O}_{4}$ nanoparticles and ferrofluid-filled photonic crystals have been widely used to realize $\mathrm{THz}$ magnetic modulators, based on magnetic induced birefringence and dichroism [22-24]. However, the THz modulation depths of $66 \%$ at $35 \mathrm{mT}, 40 \%$ at $150 \mathrm{mT}$, and $34 \%$ at $194 \mathrm{mT}$ require significantly long propagation lengths of $10 \mathrm{~mm}$ [22], $120 \mu \mathrm{m}$ [23], and $1 \mathrm{~mm}$ [24], respectively. Therefore, the development of solid-state devices is still an important topic in terms of practical THz magnetic modulators.

In this respect, magnetoresistance is an important spintronic effect for changing the conductivity response and influencing $\mathrm{THz}$ electromagnetic wave propagation. Chau et al. demonstrated that anisotropic magnetoresistance (AMR) can be used to modulate the transmission of $\mathrm{THz}$ radiation in both ensemble subwavelength Co microparticles [28] and dense micrometer-sized Co particles coated with Au microparticles [29]. Recently, we have shown that the application of a magnetic field up to $100 \mathrm{mT}$ to a giant magnetoresistive (GMR) structure induces a striking reduction of the transmission of the $\mathrm{THz}$ field by about $20 \%$. The difference in conduction by electrons with opposite spins in ferromagnetic (FM) metals was accessed precisely in a GMR stack [30]. Although the tunneling magnetoresistance (TMR) effect (TMR ratio up to around $200 \%$ ) has been probed by dc measurement statically [31] and studied within the infrared regime $(15-150 \mathrm{THz})$ using contactless reflection spectroscopy [32], a quantitative investigation of the spin-polarized electron-tunnelinginduced conductivity change in a TMR structure at $\mathrm{THz}$ frequencies has not yet been reported. In the present paper, we use single-cycle $\mathrm{THz}$ pulses to drive the spin-polarized charge across a $\mathrm{FM} / \mathrm{MgO} / \mathrm{FM}$ interface over picosecond time scales. We demonstrate that an external magnetic field (approximately $30 \mathrm{mT}$ ) tends to align the magnetization of the free FM layer with the pinned FM layer, leading to a strong attenuation of the propagating $\mathrm{THz}$ wave. We experimentally measure the increasing change of the sheet conductivity spectrum, as the magnetizations of FM layers are aligned antiparallel at zero magnetic field to coalignment under the applied magnetic field. A modulation depth as high as $60 \%$ is reached for the parallel configuration of a magnetic tunnel junction (MTJ) with a thickness of $77.45 \mathrm{~nm}$, using a magnetic field as low as $30 \mathrm{mT}$. In this work, we explore the THz TMR effect to offer a solution towards spintronic $\mathrm{THz}$ amplitude modulators.

\section{EXPERIMENTAL METHODS}

Figure 1(a) shows our MTJ multistack grown on a $\mathrm{Si}$ substrate. The MTJ multistack is $\mathrm{Ta}(5) / \mathrm{Ru}(10) / \mathrm{Ir}_{22} \mathrm{Mn}_{78}$ (10) $/ \mathrm{Co}_{75} \mathrm{Fe}_{25}$ (2.5) $/ \mathrm{Ru}(0.85) / \mathrm{Co}_{40} \mathrm{Fe}_{40} \mathrm{~B}_{20}$ (3) $/ \mathrm{MgO}(1.9) /$ free layer/ $\mathrm{Ta}(5) / \operatorname{Ru}(6)$. The numbers in parentheses indicate the thickness of each layer in nanometers. By sputter-depositing a ferromagnetic $\mathrm{Co}_{75} \mathrm{Fe}_{25}$ film on top of an antiferromagnetic layer of $\operatorname{Ir}_{22} \mathrm{Mn}_{78}$, the spin orientation of the FM thin films can be "pinned" by the exchange bias coupling between the net interfacial moment of antiferromagnetic layer and the FM layer, with a large coercive force to keep the direction of the magnetic moment in the $x$ direction, as shown in Fig. 1(a). The magnetization of the top magnet can be switched by an external magnetic field, which is called a free layer. In this work, the free layer is chosen as $\mathrm{Co}_{40} \mathrm{Fe}_{40} \mathrm{~B}_{20}(3) / \mathrm{Ru}(0.2) / \mathrm{Co}_{70.5} \mathrm{Fe}_{4.5} \mathrm{Si}_{15} \mathrm{~B}_{10}$ (30). The soft magnetic material $\mathrm{Co}_{70.5} \mathrm{Fe}_{4.5} \mathrm{Si}_{15} \mathrm{~B}_{10}$ layer has higher crystalline temperature than $\mathrm{Co}_{40} \mathrm{Fe}_{40} \mathrm{~B}_{20}$, which means that MTJs including this layer have both high TMR ratio and low coercivity of the free layer after $300-400{ }^{\circ} \mathrm{C}$ annealing. This technique has been demonstrated to provide high sensitivity and low noise for MTJs [33]. Within the free FM layer, the $\mathrm{Co}_{40} \mathrm{Fe}_{40} \mathrm{~B}_{20}$ (3) and $\mathrm{Co}_{70.5} \mathrm{Fe}_{4.5} \mathrm{Si}_{15} \mathrm{~B}_{10}$ (30) are ferromagnetically coupled via $0.2-\mathrm{nm} \mathrm{Ru}$. While within the pinned FM layer, $\mathrm{Co}_{75} \mathrm{Fe}_{25}(2.5)$ and $\mathrm{Co}_{40} \mathrm{Fe}_{40} \mathrm{~B}_{20}(3)$ are antiferromagnetically coupled via $0.85-\mathrm{nm} \mathrm{Ru}$, which is typical for obtaining strong artificial antiferromagnetic coupling. Figure 1(b) shows the magnetic hysteresis loop of our MTJ multistack, using a vibrating sample magnetometer (see Fig. S1 of Ref. [34] for details). TMR is a consequence of spin-dependent tunneling, which originates from an imbalance of the charge current carried by majority (large hollow arrows) and minority spin electrons tunneling from a ferromagnet through an insulating barrier, as shown in the inset of Fig. 1(a). For the nanometer thin FM layers with the parallel configuration of magnetizations (black arrows), the electrons with the same spin orientation with respect to the magnetization can tunnel through the $\mathrm{MgO}$ layer easily. As the magnetizations of the two FM layers are opposite, the possibility of electron tunneling between two FM layers becomes smaller, resulting in a smaller tunneling charge current compared to the case for the same directions of the magnetizations. Using the dc four-probe method in a three-dimensional Helmholtz coil system, Fig. 1(c) shows the relative resistance change of our MTJ multistack: TMR $=\left(R_{\mathrm{AP}}-R_{\mathrm{P}}\right) / R_{\mathrm{P}}=210 \%$ at $\mathbf{H}=10 \mathrm{mT}$ at room temperature, where $R_{\mathrm{P}}$ and $R_{\mathrm{AP}}$ are the tunneling resistance measured when the magnetizations are aligned in parallel and antiparallel configurations, respectively.

Our THz time-domain spectroscopy (THz-TDS) experiments are performed in a standard linear transmission configuration with the external magnetic field $\mathbf{H}$ applied in the plane of the sample. The single-cycle $\mathrm{THz}$ pulses are generated using a low-temperature (LT) GaAs photoconductive antenna with 800-nm, 100-fs Ti:sapphire laser pulses and repetition rate of $80 \mathrm{MHz}$ (MaiTai HP, SpectraPhysics). The generated $\mathrm{THz}$ pulses are collimated into 

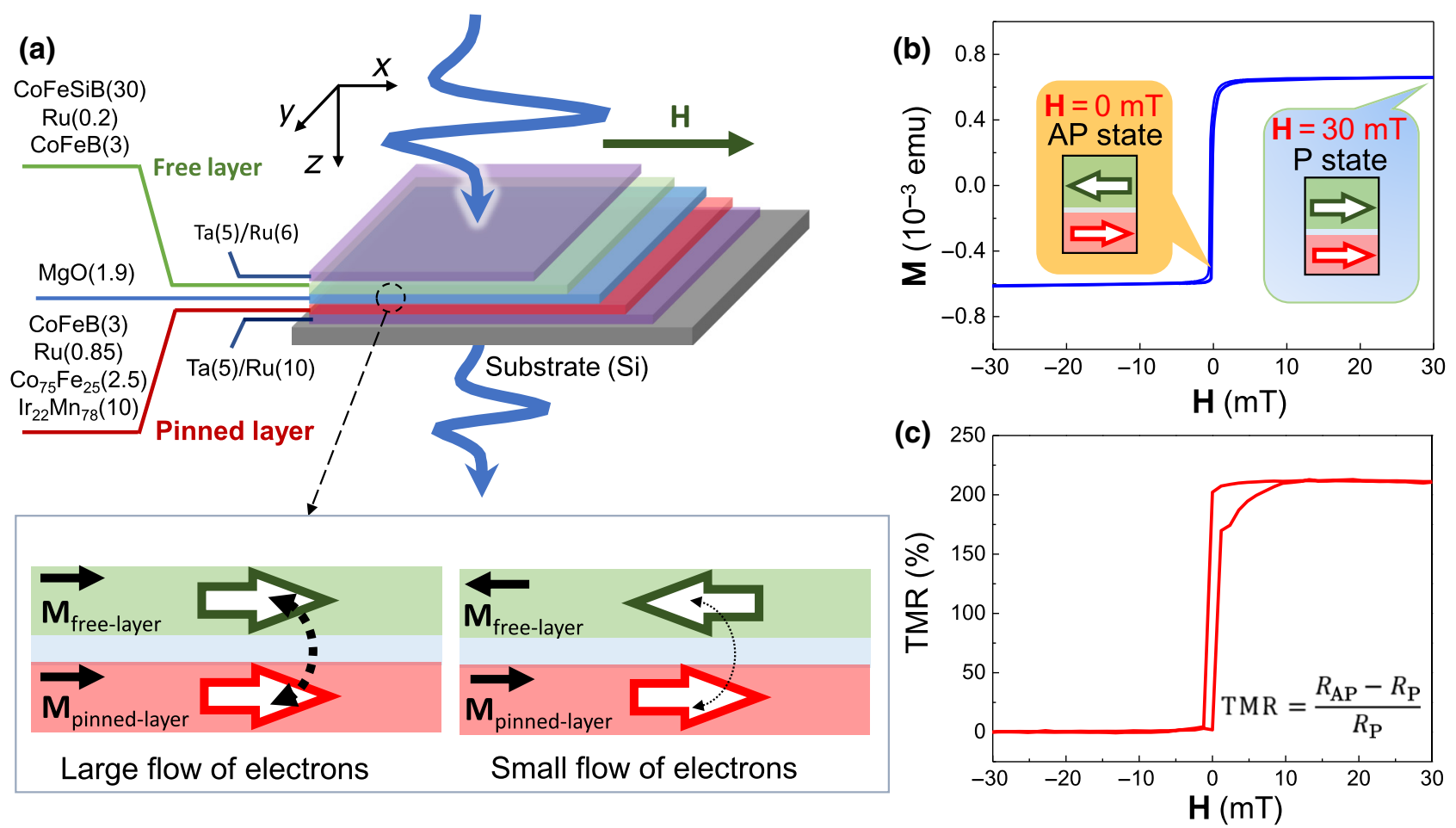

FIG. 1. (a) Schematic illustration of the THz tunneling magnetoresistance effect and the MTJ sample structure used in this work. The application of an external magnetic field, $\mathbf{H}$, leading to parallel configuration creates a large flow of electrons as compared with antiparallel configuration $(\mathbf{H}=0 \mathrm{mT})$. The statically measured (b) in-plane magnetization curve and (c) TMR ratio of the MTJ stack sample.

parallel beams by a micro silicon lens. The sample is positioned at the beam waist of the $\mathrm{THz}$ beam. The $\mathrm{THz}$ pulses are detected by photoconductive switching, which consists of a metallic dipole antenna on LT-grown GaAs. The photoinduced carriers of LT GaAs are driven by the $\mathrm{THz}$ electromagnetic field, which produces a detected current pulse. By a variable optical delay, the $\mathrm{THz}$ pulses generated at the emitter can be continuously delayed with respect to the gated detector, which allows us to temporally scan the electric field. The THz pulse spectrum covers the range $0.5-1.5 \mathrm{THz}$. The electric field of the incident $\mathrm{THz}$ pulse is linearly polarized in a horizontal plane ( $x$ axis). The weak single-cycle, sub-picosecond $\mathrm{THz}$ transients are directed normally at the sample ( $z$ axis). As shown in Fig. 1(a), the magnetic field $\mathbf{H}$ is applied in the plane of the sample ( $x$ axis), perpendicular to the propagation of the $\mathrm{THz}$ beam and parallel to the electric field polarization of the $\mathrm{THz}$ pulse. The $\mathrm{THz}$ electric field induces a time-dependent current in the sample according to its conductivity, leading to the attenuation and phase delay of the $\mathrm{THz}$ pulse as it propagated through the sample. We note that the $\mathrm{THz}$ pulse used in the present experiment is too small to influence the sample magnetization by $\mathbf{B}_{\mathrm{THz}}$, the magnetic field components of the $\mathrm{THz}$ pulse. The $\mathrm{THz}$ beam path in the spectrometer is purged with dry nitrogen in order to minimize absorption of $\mathrm{THz}$ radiation by atmospheric water vapor. All of the $\mathrm{THz}$ transmissions are averaged over five measurements at room temperature in an ambient atmosphere.

\section{EXPERIMENTAL RESULTS AND DISCUSSION}

The blue trace in Fig. 2(a) represents the typical timedomain $\mathrm{THz}$ transmitted signal $E(t)$ through the MTJ multistack with applied magnetic field $\mathbf{H}=30 \mathrm{mT}$ (along the $x$ axis), in comparison with that measured at $\mathbf{H}=0 \mathrm{mT}$ (red circles). We note a significant attenuation of $\mathrm{THz}$ peak-to-peak values of the parallel orientation $E_{\mathrm{P}}$, which is compared with $E_{\mathrm{AP}}$ at the antiparallel configuration, $\left(E_{\mathrm{AP}}-E_{\mathrm{P}}\right) / E_{\mathrm{AP}} \approx 36 \%$. Figure $2(\mathrm{~b})$ shows the instantaneous intensities of the $\mathrm{THz}$ waveforms, which are calculated from the square of the measured time-resolved $\mathrm{THz}$ electric fields in Fig. 2(a). It can also be found that these two $\mathrm{THz}$ waveforms are not accompanied by any observable temporal delays. The $\mathrm{THz}$ optical properties are essentially determined by the low-energy carrier dynamics and are especially sensitive to the electrical conductivity. This observation strongly indicates that the resistivity of the MTJ multistack depends on the relative orientation of electron spins in the pinned and free FM layers of the structure. In the absence of an applied magnetic field, the spins are aligned in an antiparallel fashion leading to a higher 

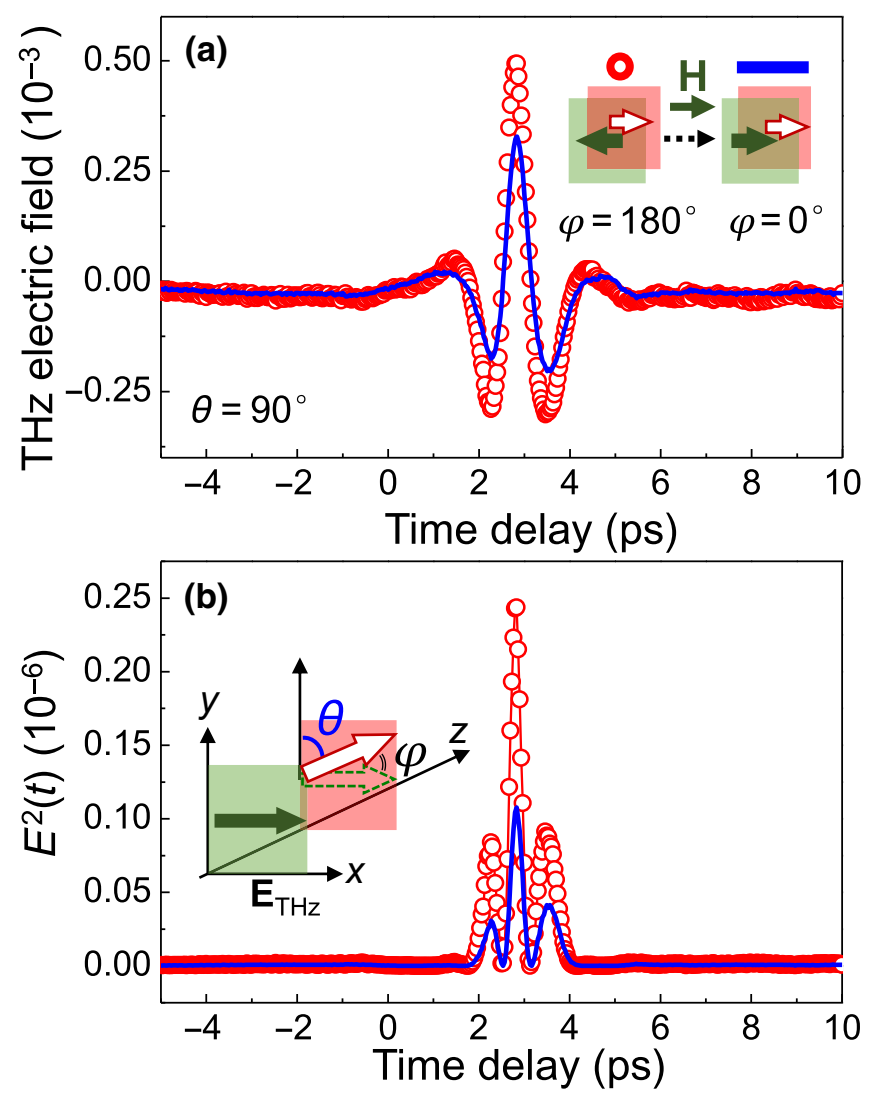

FIG. 2. (a) Time-domain $\mathrm{THz}$ waveforms $E(t)$ through the MTJ multistack for magnetizations aligned in parallel (blue line) and antiparallel (red circle) states. Inset: the orientation of the magnetic field is along the $x$ axis, which rotates $\mathbf{M}_{\text {free-layer }}$ (green arrow) along the $x$ axis. In the case of $\theta=90^{\circ}$ and $\varphi=0^{\circ}$, $\mathbf{M}_{\text {free-layer }}$ is aligned parallel to $\mathbf{M}_{\text {pinned-layer }}$ The zero time delay corresponds to an arbitrary starting position. (b) Instantaneous $\mathrm{THz}$ intensity calculated from the square of the measured electric field.

resistivity of the structure. As the spins in FM layers are coaligned in the applied magnetic field, the sample becomes more conductive, resulting in more $\mathrm{THz}$ absorption.

In our experiments, the applied magnetic field ( $x$ axis) orients $\mathbf{M}_{\text {free-layer }}$ along the $x$ axis for all cases. While it is not strong enough to align $\mathbf{M}_{\text {pinned-layer }}$ along the $x$ axis, the orientation of $\mathbf{M}_{\text {pinned-layer }}$ can be rotated by rotating the sample. We define the azimuthal angle $\theta$ as the angle between the magnetization of the pinned layer $\mathbf{M}_{\text {pinned-layer }}$ and the $y$ axis. In addition, the relative angle $\varphi$ is defined by the angle between $\mathbf{M}_{\text {free-layer }}$ and $\mathbf{M}_{\text {pinned-layer }}$, as shown in the inset of Fig. 2(b). By rotating the azimuthal angles $\theta=180^{\circ}, 270^{\circ}$, and $360^{\circ}\left(0^{\circ}\right)$, we obtain the $\mathrm{THz}$ waveforms $E(t)$ with different relative angles $\varphi=90^{\circ}, 180^{\circ}$, and $270^{\circ}$ as the blue curves shown in Figs. 3(a)-3(c), respectively. Firstly, they are compared to $E(t)$ transmitted through the MTJ multistack measured at $\mathbf{H}=0 \mathrm{mT}$ (red circles). The relative directions of $\mathbf{M}_{\text {free-layer }}$ and $\mathbf{M}_{\text {pinned-layer }}$ are shown in the insets of Fig. 3. As shown in Fig. 3(b), for $\theta=270^{\circ}$ and $\varphi=180^{\circ}$, the $\mathrm{THz}$ waveform is almost the same as that measured at zero magnetic field antiparallel orientation, owing to the magnetic field not changing the orientation of $\mathbf{M}_{\text {free-layer }}$. In the cases of $\theta=180^{\circ}, \varphi=90^{\circ}$ [Fig. 3(a)] and $\theta=0^{\circ}, \varphi=270^{\circ}$ [Fig. 3(c)], $\mathbf{M}_{\text {free-layer }}$ is oriented perpendicular to $\mathbf{M}_{\text {pinned-layer }}$, the transmitted $\mathrm{THz}$ amplitudes (blue curves) are smaller than that measured at zero magnetic field antiparallel orientation (red circles), while they are larger than that measured at $\varphi=0^{\circ}$ [blue curve in Fig. 2(a)]. From our experimental results, we can see that the change of the magnetic configurations between $\mathbf{M}_{\text {free-layer }}$ and $\mathbf{M}_{\text {pinned-layer within }}$ the MTJ has a modulating effect on the propagating $\mathrm{THz}$ electromagnetic pulses.

As the red circles show in Figs. 3(a)-3(c), the incident $\mathrm{THz}$ wave with the electric field polarized parallel to $\mathbf{M}_{\text {pinned-layer }}$ (i.e., $\theta=90^{\circ}, 270^{\circ}$ ) undergoes an increased absorption. While the $\mathrm{THz}$ electric field polarized orthog-

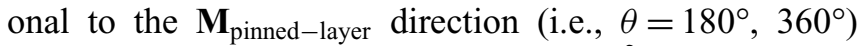
undergoes a reduced attenuation. $I=\int E^{2}(t) d t$ is used to characterize the intensity of $\mathrm{THz}$ waveform transmitted through the sample with ultrafast time resolution. The data

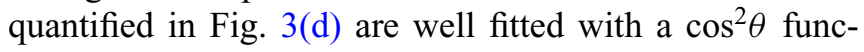
tion by the solid line, which can be attributed to AMR. AMR originates from the scattering anisotropy due to the spin-orbit interaction. The $\mathrm{THz}$ pulse transmission through the MTJ sample is influenced by magnetically varying the resistivity. We find that the modulation of the $\mathrm{THz}$ intensity by AMR is around $16 \%$. Note that for the sufficiently thin nanometer layers used in our MTJ, we do not observe any reshaping and delay of the $\mathrm{THz}$ waveforms, which are observed in subwavelength-size Co particles with sample length ranging from 2 to $9 \mathrm{~mm} \mathrm{[28]} \mathrm{and} \mathrm{Co} / \mathrm{Au}$ bimetallic particles with sample length of $3 \mathrm{~mm}$ [29].

The further implications between the magnetization configuration of the MTJ sample and $\mathrm{THz}$ transmitted intensity are emphasized in Fig. 3(e), which are strongly dependent on the rotation angle $\varphi$. It should be noted that the dc resistance of the MTJ multistack can be expressed as $R(\varphi)=(1 / 2)\left(R_{\mathrm{AP}}+R_{\mathrm{P}}\right)-(1 / 2)\left(R_{\mathrm{AP}}-R_{\mathrm{P}}\right) \cos \varphi$. Since the $\mathrm{THz}$ transmission is correlated to the resistance, it is reasonable to expect that the intensity of $\mathrm{THz}$ transmission and the dc resistance share the same $\cos \varphi$ dependence. Thus

$$
I(\varphi)=\frac{1}{2}\left(I_{\mathrm{AP}}+I_{\mathrm{P}}\right)-\frac{1}{2}\left(I_{\mathrm{AP}}-I_{\mathrm{P}}\right) \cos \varphi,
$$

where $I_{\mathrm{AP}}$ and $I_{\mathrm{P}}$ are the intensity of $\mathrm{THz}$ waveforms for parallel and antiparallel states, respectively. Obviously, Fig. 3(e) shows that $I(\varphi)$ follows mainly the $\cos \varphi$ dependence, which again provides strong and direct evidence of TMR. Actually, we can calculate $I(\varphi)$ at different $(\varphi)$ using the measured values of $I_{\mathrm{AP}}$ and $I_{\mathrm{P}}$. Besides TMR, the experimental measured $\mathrm{THz}$ transmission can also be determined by several other contributions: (1) intrinsic 


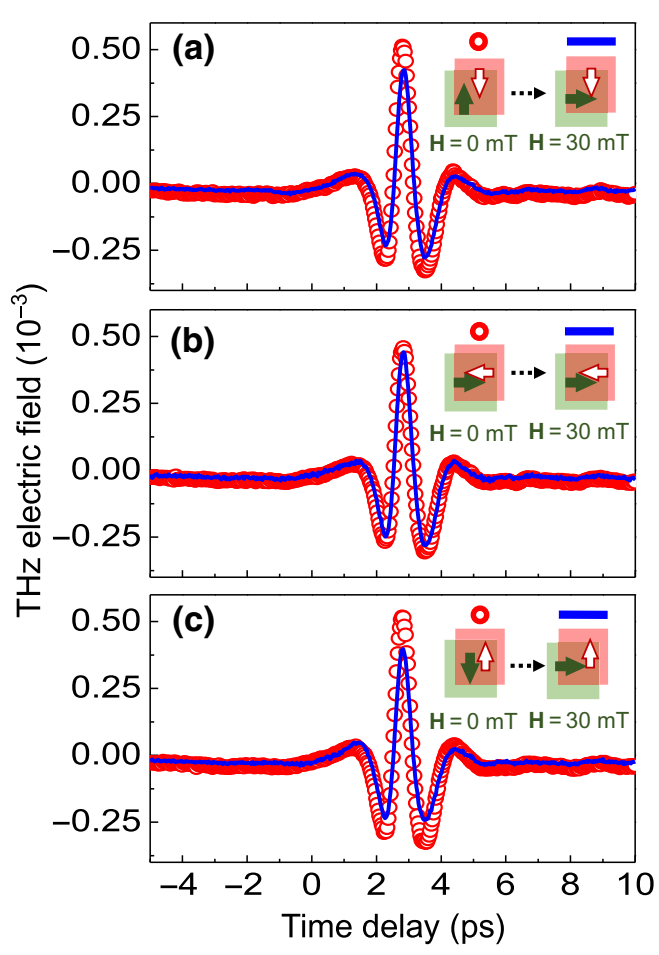

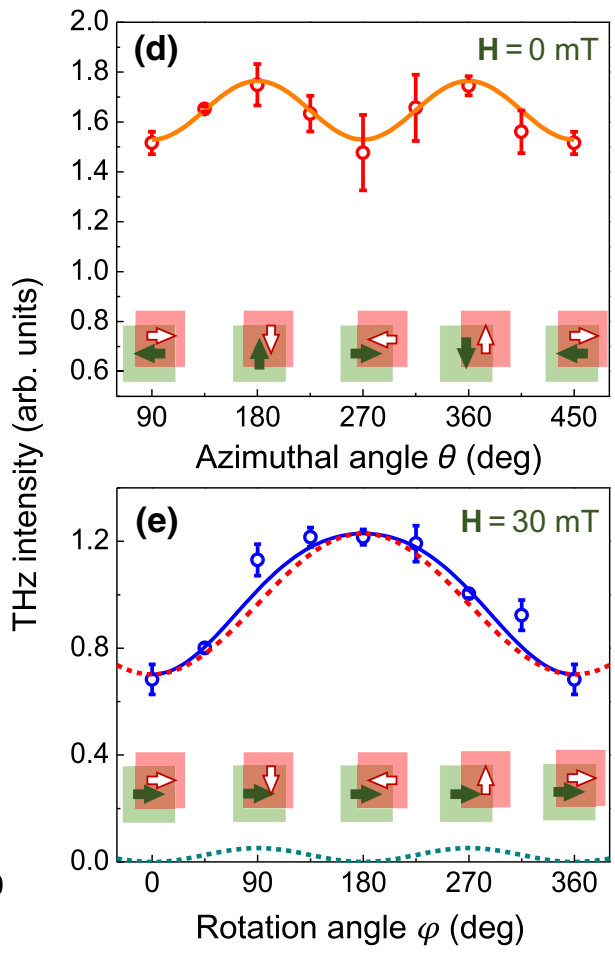

FIG. 3. Time-domain $\mathrm{THz}$ waveforms $E(t)$ through the MTJ multistack measured at $\mathbf{H}=0 \mathrm{mT}$ (red circles) and at $\mathbf{H}=30 \mathrm{mT}$ applied along the $x$ axis (blue traces) for selected azimuthal angles of (a) $\theta=180^{\circ}$, (b) $\theta=270^{\circ}$, and (c) $\theta=360^{\circ}$. (d) THz intensities $I=\int E^{2}(t) d t$ are calculated from the $\mathrm{THz}$ waveforms transmitted through the MTJ versus $\theta$ in the configuration of antiparallel state. The solid line shows a $\cos ^{2} \theta$ fit. (e) As $\mathbf{M}_{\text {free-layer }}$ is fixed along the $x$ axis, $\varphi$ is dependent on I. The full fitting (blue line) includes both the AMR (green dotted line) and TMR (red dashed line) contributions. resistance of the Si substrate, (2) nonmagnetic interface resistance between the metallic layers, and (3) AMR. Indeed, the first two terms do not depend on the rotation angle $\varphi$. Therefore, we simultaneously consider both AMR and TMR contributions to the entirety of measured data. The dotted line and the dashed line show independently AMR with $\cos ^{2} \theta$ dependence and TMR with $\cos \varphi$ dependence, respectively. We can see that TMR plays a significant role in the observed effect under an applied magnetic field.

One could argue that the magnitudes of magnetizations of free layer and pinned layer are not exactly the same, which builds up a net magnetization of the MTJ multistack. Therefore, in such a ferromagnetic anisotropic material, the role of magnetization-induced birefringence and dichroism in the propagating $\mathrm{THz}$ wave is required to be examined [22]. It should be mentioned that, as the magnetization is parallel (orthogonal) to the polarization plane of a propagating $\mathrm{THz}$ wave, linear (circular) birefringence can be induced, corresponding to the Voigt (Faraday) configuration in the $\mathrm{THz}$ regime. There have been a series of demonstrations of the Faraday rotation in two-dimensional electron gases, $\mathrm{HgTe}$ thin films, topological insulators, and high- $T_{c}$ superconductors [35-38]. Because the THz polarization can be modulated by the Faraday effect, when either spatial inversion or time reversal symmetry in the materials is breaking. We test the polarization of transmitted $\mathrm{THz}$ waveforms using time-domain $\mathrm{THz}$ polarimetry $[39,40]$ (see Fig. S2 of Ref. [34] for details). Figure S2 shows the three-dimensional trajectory for the transmitted $\mathrm{THz}$ electric fields through the MTJ multistack with and without a magnetic field. We find that the magnetic field modulates the $\mathrm{THz}$ transmittance, while the polarization state of the THz pulse after it passes through the MTJ sample does not show any changes, relative to the incident one. These experimental results confirm again that due to the TMR effect, the magnetically varying conductivity causes a strong spin-dependent THz transmission modulation. This means that the $\mathrm{THz}$ propagation properties in our metallic sample mainly depend on the electronic transports on the Fermi level.

For a quantitative analysis, we Fourier-transform the transmitted $\mathrm{THz}$ signals. Figures 4(a)-4(c) display the frequency-dependent $\mathrm{THz}$ spectra $E_{\varphi}(\omega / 2 \pi)$ transmitted through the ensemble TMR multistack on the Si substrate for the antiparallel state $\left(\varphi=180^{\circ}\right)$, perpendicular state $\left(\varphi=90^{\circ}\right)$, and parallel state $\left(\varphi=0^{\circ}\right)$. We evaluate the efficiency of the modulation process using the energy spectral density modulation depth [22]:

$$
M_{\varphi}(\omega)=\frac{\left|E_{\mathrm{AP}}(\omega)\right|^{2}-\left|E_{\varphi}(\omega)\right|^{2}}{\left|E_{\mathrm{AP}}(\omega)\right|^{2}} .
$$

Figure 4(d) shows the frequency-resolved modulation depth for the MTJ sample at three given states: $\varphi=0^{\circ}$, $90^{\circ}, 180^{\circ}$. In the case of the parallel state $\left(\varphi=0^{\circ}\right)$, $M_{\varphi=0^{\circ}}(\omega / 2 \pi)$ is found to be around $60 \%$ over the frequency range $0.6-1.2 \mathrm{THz} . M_{\varphi}(\omega / 2 \pi)$ shows a very weak $\mathrm{THz}$ frequency dependence in all three cases. In Fig. 4(e), the symbols show the dependency of the measured $\mathrm{THz}$ 


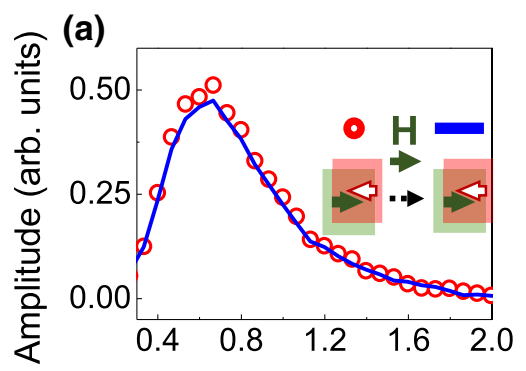

(b)

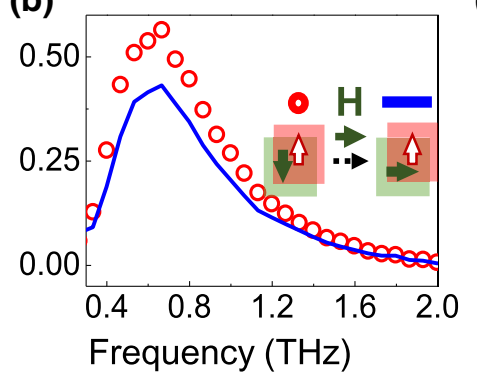

(c)

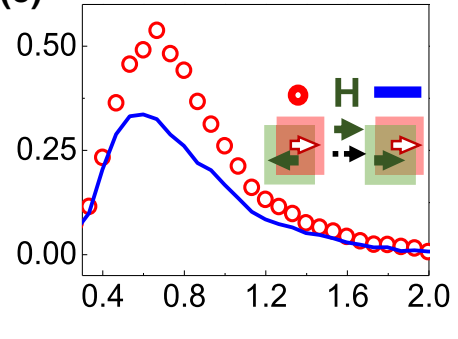

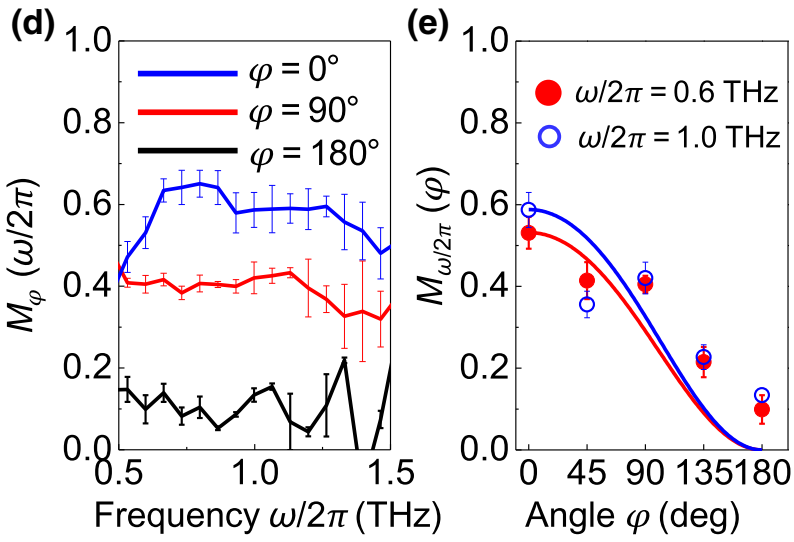

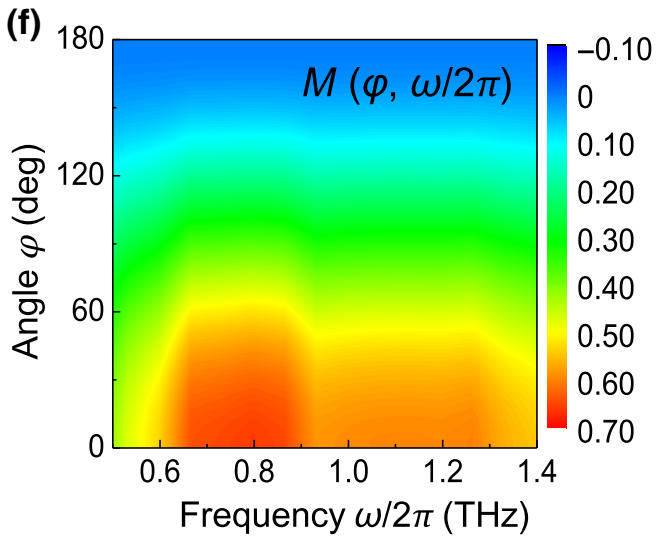

FIG. 4. Example amplitude spectra of the measured $\mathrm{THz}$ time traces under different magnetization configurations. (a) Antiparallel state $\left(\theta=270^{\circ}, \varphi=180^{\circ}\right)$, (b) perpendicular state $\left(\theta=0^{\circ}, \varphi=90^{\circ}\right)$, and (c) parallel state $\left(\theta=90^{\circ}, \varphi=0^{\circ}\right)$. For the Fourier transformation, the $\mathrm{THz}$ signal in the time domain $-5 \mathrm{ps}<t<10 \mathrm{ps}$ is used, which limits the frequency resolution to be $0.26 \mathrm{meV}$. (d) The frequency-dependent modulation depth of the THz waveforms $M_{\varphi}(\omega / 2 \pi)$ calculated using Eq. (2) for $\varphi=0^{\circ}, 90^{\circ}$, and $180^{\circ}$. (e) The angle-dependent modulation depth $M_{\omega / 2 \pi}(\varphi)$ for $\omega / 2 \pi=0.6 \mathrm{THz}$ and $1.0 \mathrm{THz}$. (f) The calculated $M(\varphi, \omega / 2 \pi)$ as functions of frequencies and angles, using the experimental $E_{\mathrm{P}}(\omega / 2 \pi)$ and $E_{\mathrm{AP}}(\omega / 2 \pi)$ as input.

modulation depth on the relative angle $\varphi$, at two selected frequencies of $\omega / 2 \pi=0.6$ and $1.0 \mathrm{THz}$. Using Eqs. (1) and (2), we calculate the $\varphi$ dependence of $\mathrm{THz}$ waveform modulation depth $M_{\omega / 2 \pi}(\varphi)$, as shown by the solid lines in Fig. 4(e). Excellent agreement between the measured data and the model is observed. Therefore, the modulation of THz signals through the MTJ can be readily calculated only using the experimentally determined $E_{\mathrm{P}}(\omega / 2 \pi)$ and $E_{\mathrm{AP}}(\omega / 2 \pi)$, as illustrated in the mapping of $M(\varphi, \omega / 2 \pi)$ in Fig. 4(f). In this regard, our results indicate the potential for heat- and contact-free TMR readout using THz transients. The maximum modulation depth obtained in our MTJ sample (approximately 60\%) is comparable to that of the $\mathrm{THz}$ magnetic modulator based on $\mathrm{Fe}_{3} \mathrm{O}_{4}$ nanoparticles $(66 \%)$ [22]. It should be noted that the thickness of our MTJ sample is $4-5$ orders of magnitude less than that of the magnetically clustered particles [22,28,29].

Analogous to electrically driven current in the TMR system, the $\mathrm{THz}$ pulse has been demonstrated to drive the spin-polarized current optically due to the different conductivities of majority and minority spin electrons inside the FM layer [30]. We consider that the electron wave function is delocalized across multiple layers of the MTJ structure and assume a Fermi velocity of $v_{F} \sim 10^{6} \mathrm{~m} / \mathrm{s}$ and momentum scattering time range of $20-50$ fs. The average mean free path of the electrons is estimated to be $\lambda_{e}=v_{F} \times \tau \sim 20-50 \mathrm{~nm}$, of the order of our whole MTJ structure of $77.45 \mathrm{~nm}$. Chau et al. also mentioned that in the $\mathrm{THz}$ regime, the skin depth of the current is around $100 \mathrm{~nm}$ within bimetallic microparticles [29]. Actually, due to the Heisenberg uncertainty principle, the spatial localization of an electron within one thin metallic layer can result in a significant component of its momentum in the direction perpendicular to the plane of the layer. Therefore, the effective medium approach can be applied to describe the electron conduction in TMR multilayers. It should be also noted that our weak $\mathrm{THz}$ transients are not sufficient to induce any electronic or magnetic nonlinearity in the sample, providing for a linear conductivity measurement.

Now we present differential measurements to yield the spin-polarized electron-tunneling-induced complex sheet conductivity $\Delta \sigma(\omega)$ using the thin-film equation $E_{\varphi}(\omega) / E_{\mathrm{AP}}(\omega)=(n+1) /\left[n+1+\mathrm{Z}_{0} \Delta \sigma(\omega)\right] \quad[5,6]$, where the measured $E_{\mathrm{AP}}(\omega)$ (highest resistive case) is taken as a reference and $E_{\varphi}(\omega)$ is taken as a sample signal. $n=3.2$ is the measured effective real $\mathrm{THz}$ refractive index of the whole sample structure $[41,42]$ (see Fig. S3 


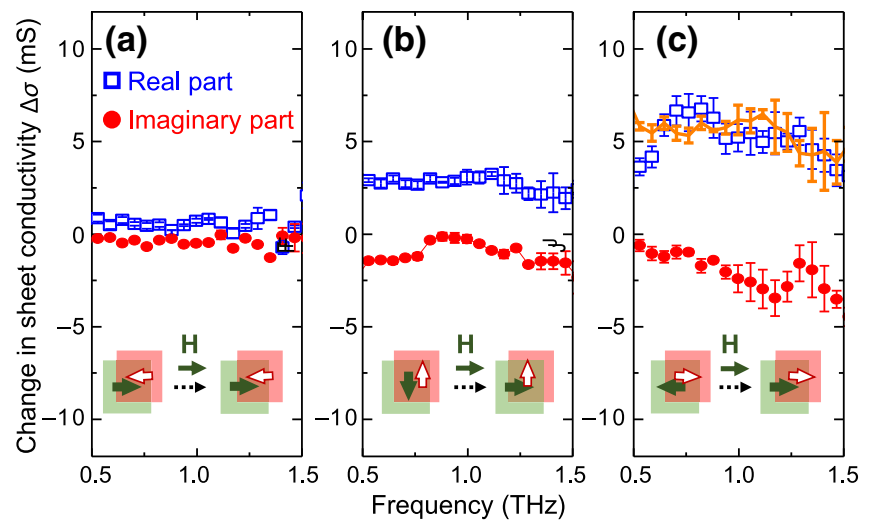

FIG. 5. The magnetic-field-induced change in sheet conductivity $\Delta \sigma$ of the MTJ multistack for three representative magnetization configurations, calculated from the Fourier transform of the time-domain electric field $E_{\varphi}$ through the sample and $E_{\mathrm{AP}}$ for the reference. Real and imaginary parts of $\Delta \sigma$ for (a) antiparallel state $\left(\theta=270^{\circ}, \varphi=180^{\circ}\right)$, (b) perpendicular state $\left(\theta=0^{\circ}\right.$, $\left.\varphi=90^{\circ}\right)$, and (c) parallel state $\left(\theta=90^{\circ}, \varphi=0^{\circ}\right)$.

of Ref. [34] for details) and $Z_{0}=377 \Omega$ is the impedance of free space. $\Delta \sigma(\omega)=\sigma_{\varphi}(\omega)-\sigma_{\mathrm{AP}}(\omega)$ is the modulation of the frequency-sensitive sheet conductivity owing to the change of electron tunneling in the MTJ multistack.

Figure 5 shows the measured real component $\Delta \sigma_{1}$ $(\omega / 2 \pi)$ (square symbols) and imaginary component $\Delta \sigma_{2}(\omega / 2 \pi)$ (circle symbols) within the THz spectral range of $0.5-1.5 \mathrm{THz}$. It is important to see that $\Delta \sigma_{1}(\omega / 2 \pi)$ is markedly increased, as $\varphi$ changes from $180^{\circ}$ (antiparallel state), to $90^{\circ}$ (perpendicular state), and finally to $0^{\circ}$ (parallel state), indicating that the electron tunneling is increased. We note that the conductivity of the MTJ multistack depends on the angle between the respective magnetization of two magnetic layers [43]:

$$
\sigma_{\mathrm{dc}}=\sigma_{0}\left(1+P \times P^{*} \cos \varphi\right)
$$

where $\sigma_{0}$ is the mean conductance and $P$ and $P^{*}$ are the effective spin polarization coefficients of the ferromagnetic-barrier coupling, including the simple spin polarization of the free and the pinned FM layers, as well as the contribution from the interface [43]. The angular dependence as shown by Eq. (3) is verified by dc measurements. However, it is not demonstrated in the $\mathrm{THz}$ frequency range. According to Eq. (3), $\Delta \sigma_{\varphi=90^{\circ}}(\omega / 2 \pi)=\sigma_{\varphi=90^{\circ}}(\omega / 2 \pi)-$ $\sigma_{\mathrm{AP}}(\omega / 2 \pi)=\sigma_{0}(\omega / 2 \pi) P \times P^{*}$, while $\Delta \sigma_{\varphi=0^{\circ}}(\omega / 2 \pi)=$ $\sigma_{\varphi=0^{\circ}}(\omega / 2 \pi)-\sigma_{\mathrm{AP}}(\omega / 2 \pi)=2 \sigma_{0}(\omega / 2 \pi) P \times P^{*}$. It can be noted that $2 \times \Delta \sigma_{\varphi=90^{\circ}}(\omega / 2 \pi)=\Delta \sigma_{\varphi=0^{\circ}}(\omega / 2 \pi)$. The orange line in Fig. 5(c) is the calculated result of the measured $\Delta \sigma_{\varphi=90^{\circ}}(\omega / 2 \pi)$ after multiplication by 2 , which is consistent with the measured $\Delta \sigma_{\varphi=0^{\circ}}(\omega / 2 \pi)$. Our experimental findings demonstrate a good agreement with the TMR theoretical prediction over the wide THz bandwidth.
Finally, we note here that for a prospective $\mathrm{THz}$ modulation device $[10,11]$, on the one hand, a fast modulation speed is required. In principle, the magnetic fields of $\mathrm{THz}$ pulses radiating from high-dielectric-constant lithium niobite, large-size ZnTe, and two-color laser filaments are enough to act as ultrafast magnetic pulses to switch $\mathbf{M}_{\text {free-layer }}$ within picosecond time scales [44]. If the magnetic field $\mathbf{B}_{\mathrm{THz}}$ of $\mathrm{THz}$ pulse results in a reorientation of $\mathbf{M}_{\text {free-layer }}$ through the Landau-Lifschitz-Gilbert formalism, the resistance of the MTJ changes through the TMR effect during the pulse duration. We will leave the investigation of the $\mathrm{THz}$ transmission depending on the strength of $\mathrm{THz}$ input pulse to future work.

On the other hand, we believe that the MTJ-based THz modulation demonstrated here can be integrated with the concept of spintronic THz emitters (such as W/FM/Pt [45] or $\mathrm{FM} / \mathrm{Ag} / \mathrm{Bi}[46,47]$ heterostructures). The noncollinear configuration of magnetizations in the synthetic spintronic structures will be useful for further designs of $\mathrm{THz}$ emitters with tunable amplitudes and polarizations [48,49].

\section{CONCLUSION}

In summary, we demonstrate $\mathrm{THz}$ magnetic modulation using the THz TMR effect, which is governed by spin-dependent electron tunneling on an ultrafast (sub-) picosecond time scale. The MTJ-based THz modulation not only combines a high modulation depth and low magnetic field requirements, but also shows flexibility given by the tunability of $\mathrm{THz}$ conductivity in response to the relative magnetization orientations. We believe that the modulation amplitude can be further improved by optimizing structures and parameters of the FM material and insulating barrier. As the magnetization can be switched (or quenched) by ultrashort $\mathrm{THz}$ or laser pulses, it should be possible to achieve a rather high-speed modulation device with operating frequency up to THz. Moreover, the MTJ can be further integrated with other metamaterials and spintronic $\mathrm{THz}$ emitters, which will be useful for further designs of unique $\mathrm{THz}$ functional devices.

\section{ACKNOWLEDGMENTS}

We are grateful to Tobias Kampfrath for useful discussions. X.F.H. is grateful for the financial support from the National Key Research and Development Program of China (Grant No. 2017YFA0206200). This work is supported by the National Natural Science Foundation of China (Grant Nos. 61975110, 11604202, $11674213,61735010)$. This work is supported in part by the National Natural Science Foundation of China (Grant No. 61722111), the 111 Project (Grant No. D18014), the International Joint Lab Program supported by Science and Technology Commission Shanghai Municipality (Grant No. 17590750300), and the key project 
supported by Science and Technology Commission Shanghai Municipality (Grant No. YDZX20193100004960). Z.J. thanks Shanghai Municipal Education Commission (Young Eastern Scholar Grant No. QD2015020), Science and Technology Commission of Shanghai Municipality (Shanghai Rising-Star Program Grant No. 18QA1401700), and Shanghai Educational Development Foundation (Chen Guang project Grant No. 16CG45). C.H.W. appreciates financial support from Youth Innovation Promotion Association, CAS (Grant No. 2020008).

[1] M. Tonouchi, Cutting-edge terahertz technology, Nat. Photonics 1, 97 (2007).

[2] B. Ferguson and X. C. Zhang, Materials for terahertz science and technology, Nat. Mater. 1, 26 (2002).

[3] T. Kleine-Ostmann and T. Nagatsuma, A review on terahertz communications research, J. Infrared. Millim. Te. 32, 143 (2011).

[4] S. Koenig, D. Lopez-Diaz, J. Antes, F. Boes, R. Henneberger, A. Leuther, A. Tessmann, R. Schmogrow, D. Hillerkuss, R. Palmer, T. Zwick, C. Koos, W. Freude, O. Ambacher, J. Leuthold, and I. Kallfass, Wireless sub-THz communication system with high data rate, Nat. Photonics 7, 977 (2013).

[5] P. U. Jepsen, D. G. Cooke, and M. Koch, Terahertz spectroscopy and imaging-modern techniques and applications, Laser Photonics Rev. 5, 124 (2011).

[6] R. Ulbricht, E. Hendry, J. Shan, T. F. Heinz, and M. Bonn, Carrier dynamics in semiconductors studied with timeresolved terahertz spectroscopy, Rev. Mod. Phys. 83, 543 (2011).

[7] P. H. Siegel, Terahertz technology in biology and medicine, IEEE T. Microw. Theory. 52, 2438 (2004).

[8] Y. Peng, C. J. Shi, Y. M. Zhu, M. Gu, and S. L. Zhuang, Terahertz spectroscopy in biomedical field: A review on signal-to-noise ratio improvement, PhotoniX. 1, 12 (2020).

[9] J. F. Federici, B. Schulkin, F. Huang, D. Gary, R. Barat, F. Oliveira, and D. Zimdars, THz imaging and sensing for security applications - explosives, weapons and drugs, Semicond. Sci. Technol. 20, S266 (2005).

[10] M. Rahm, J.-S. Li, and W. Padilla, THz wave modulators: A brief review on different modulation techniques, J. Infrared. Millim. Te. 34, 1 (2013).

[11] L. Wang, Y. X. Zhang, X. Q. Guo, T. Chen, H. J. Liang, X. L. Hao, X. Hou, W. Kou, Y. C. Zhao, T. C. Zhou, S. X. Liang, and Z. Q. Yang, A review of THz modulators with dynamic tunable metasurfaces, Nanomaterials. 9, 965 (2019).

[12] P. Weis, J. L. Garcia-Pomar, M. Höh, B. Reinhard, A. Brodyanski, and M. Rahm, Spectrally wide-band terahertz wave modulator based on optically tuned graphene, ACS Nano 6, 9118 (2012).

[13] B. Sensale-Rodriguez, R. Yan, M. M. Kelly, T. Fang, K. Tahy, W. S. Hwang, D. Jena, L. Liu, and H. G. Xing, Broadband graphene terahertz modulators enabled by intraband transitions, Nat. Commun. 3, 780 (2012).
[14] Z. F. Chen, X. Q. Chen, L. Tao, K. Chen, M. Z. Long, X. D. Liu, K. Y. Yan, R. I. Stantchev, E. Pickwell-MacPherson, and J.-B. Xu, Graphene controlled brewster angle device for ultra broadband terahertz modulation, Nat. Commun. 9 , 4909 (2018).

[15] R. Kowerdziej, M. Olifierczuk, and J. Parka, Thermally induced tunability of a terahertz metamaterial by using a specially designed nematic liquid crystal mixture, Opt. Express 26, 2443 (2018).

[16] L. Cheng, Z. M. Jin, Z. W. Ma, F. H. Su, Y. Zhao, Y. Z. Zhang, T. Y. Su, Y. Sun, X. L. Xu, Z. Meng Y, C. Bian, and Z. G. Sheng, Mechanical terahertz modulation based on single-layered graphene, Adv. Opt. Mater. 6, 1700877 (2018).

[17] T. Kampfrath, M. Battiato, P. Maldonado, G. Eilers, J. Nötzold, S. Mährlein, V. Zbarsky, F. Freimuth, Y. Mokrousov, S. Blügel, M. Wolf, I. Radu, P. M. Oppeneer, and M. Münzenberg, Terahertz spin current pulses controlled by magnetic heterostructures, Nat. Nanotechnol. 8, 256 (2013).

[18] T. Seifert, et al., Efficient metallics spintronic emitters of ultrabroadband terahertz radiation, Nat. Photonics 10, 483 (2016).

[19] T. J. Huisman, R. V. Mikhaylovskiy, J. D. Costa, F. Freimuth, E. Paz, J. Ventura, P. P. Freitas, S. Blügel, Y. Mokrousov, Th. Rasing, and A. V. Kimel, Femtosecond control of electric currents in metallic ferromagnetic heterostructures, Nat. Nanotech. 11, 455 (2016).

[20] K. Yamaguchi, M. Nakajima, and T. Suemoto, Coherent Control of Spin Precession Motion with Impulsive Magnetic Fields of Half-Cycle Terahertz Radiation, Phys. Rev. Lett. 105, 237201 (2010).

[21] X. W. Li, M. Bamba, N. Yuan, Q. Zhang, Y. Zhao, M. Xiang, K. Xu, Z. M. Jin, W. Ren, G. H. Ma, S. X. Cao, D. Turchinovich, and J. Kono, Observation of dicke cooperativity in magnetic interactions, Science 361, 794 (2018).

[22] M. Shalaby, M. Peccianti, Y. Ozturk, I. Al-Naib, C. P. Hauri, and R. Morandotti, Terahertz magnetic modulator based on magnetically clustered nanoparticles, Appl. Phys. Lett. 105, 151108 (2014).

[23] F. Fan, S. Chen, W. Lin, Y. P. Miao, S. J. Chang, B. Liu, X. H. Wang, and L. Lin, Magnetically tunable terahertz magnetoplasmons in ferrofluid-filled photonic crystals, Appl. Phys. Lett. 103, 161115 (2013).

[24] M. Liu, L. Y. Xiong, X. Yu, S. L. He, B. Zhang, and J. L. Shen, Magnetically controlled terahertz modulator based on $\mathrm{Fe}_{3} \mathrm{O}_{4}$ nanoparticle ferrofluids, J. Phys. D: Appl. Phys. 51, 105003 (2018).

[25] F. Fan, S. Chen, and S. J. Chang, A review of magnetooptical microstructure devices at terahertz frequencies, IEEE J. Sel. Top. Quantum Electron. 23, 8500111 (2017).

[26] R. R. Subkhangulov, R. V. Mikhaylovskiy, A. K. Zvezdin, V. V. Kruglyak, Th. Rasing, and A. V. Kimel, Terahertz modulation of the Faraday rotation by laser pulses via the optical kerr effect, Nat. Photonics 10, 111 (2016).

[27] Q. Mu, F. Fan, S. Chen, S. Xu, C. Xiong, X. Zhang, X. Wang, and S. Chang, Tunable magneto-optical polarization device for terahertz waves based on InSb and its plasmonic structure, Photonics Res. 7, 325 (2019). 
[28] K. J. Chau and A. Y. Elezzabi, Photonic Anisotropic Magnetoresistance in Dense Co Particle Ensembles, Phys. Rev. Lett. 96, 033903 (2006).

[29] K. J. Chau, M. Johnson, and A. Y. Elezzabi, Electronspin-dependent Terahertz Light Transport in SpintronicPlasmonic Media, Phys. Rev. Lett. 98, 133901 (2007).

[30] Z. M. Jin, A. Tkach, F. Casper, V. Spetter, H. Grimm, A. Thomas, T. Kampfrath, M. Bonn, M. Kläui, and D. Turchinovich, Accessing the fundamentals of magnetotransport in metals with terahertz probes, Nat. Phys. 11, 761 (2015).

[31] S. Yuasa, T. Nagahama, A. Fukushima, Y. Suzuki, and K. Ando, Giant room-temperature magnetoresistance in single-crystal $\mathrm{Fe} / \mathrm{MgO} / \mathrm{Fe}$ magnetic tunnel junctions, Nat. Mater. 3, 868 (2004).

[32] M. Vopsaroiu, D. Bozec, J. A. D. Matthew, S. M. Thompson, C. H. Marrows, and M. Perez, Contactless magnetoresistance studies of $\mathrm{Co} / \mathrm{Cu}$ multilayers using the infrared magnetorefractive effect, Phys. Rev. B 70, 214423 (2004).

[33] L. Huang, Z. H. Yuan, B. S. Tao, C. H. Wan, P. Guo, Q. T. Zhang, L. Yin, J. F. Feng, T. Nakano, H. Naganuma, H. F. Liu, Y. Yan, and X. F. Han, Noise suppression and sensitivity manipulation of magnetic tunnel junction sensors with soft magnetic $\mathrm{Co}_{70.5} \mathrm{Fe}_{4.5} \mathrm{Si}_{15} \mathrm{~B}_{10}$ layer, J. Appl. Phys. 122, 113903 (2017).

[34] See Supplemental Material at http://link.aps.org/supple mental/10.1103/PhysRevApplied.14.014032 for magnetic hysteresis loop, polarization of the transmitted $\mathrm{THz}$ waveforms, and THz real refractive index of the MTJ multistack on the silicon substrate.

[35] A. V. Stier, C. T. Ellis, J. Kwon, H. Xing, H. Zhang, D. Eason, G. Strasser, T. Morimoto, H. Aoki, H. Zeng, B. D. McCombe, and J. Cerne, Terahertz Dynamics of a Topologically Protected State: Quantum Hall Effect Plateaus Near the Cyclotron Resonance of a two-Dimensional Electron gas, Phys. Rev. Lett. 115, 247401 (2015).

[36] A. M. Shuvaev, G. V. Astakhov, A. Pimenov, C. Brüne, H. Buhmann, and L. W. Molenkamp, Giant Magneto-Optical Faraday Effect in HgTe Thin Films in the Terahertz Spectral Range, Phys. Rev. Lett. 106, 107404 (2011).

[37] L. Wu, M. Salehi, N. Koirala, J. Moon, S. Oh, and N. Armitage, Quantized Faraday and kerr rotation and axion electrodynamics of a 3D topological insulator, Science 354, 1124 (2016).

[38] Y. Lubashevsky, L. D. Pan, T. Kirzhner, G. Koren, and N. P. Armitage, Optical Birefringence and Dichroism of Cuprate
Superconductors in the THz Regime, Phys. Rev. Lett. 112, 147001 (2014).

[39] O. Morikawa, A. Quema, S. Nashima, H. Sumikura, T. Nagashima, and M. Hangyo, Faraday ellipticity and Faraday rotation of a doped-silicon wafer studied by terahertz time-domain spectroscopy, J. Appl. Phys. 100, 033105 (2006).

[40] M. Nakajimal, A. Namai, S. Ohkoshi, and T. Suemoto, Ultrafast time domain demonstration of bulk magnetization precession at zero magnetic field ferromagnetic resonance induced by terahertz magnetic field, Opt. Express 18, 18260 (2010).

[41] J. Neu and C. A. Schmuttenmaer, Tutorial: An introduction to terahertz time domain spectroscopy (THz-TDS), J. Appl. Phys. 124, 231101 (2018).

[42] M. Hangyo, M. Tani, and T. Nagashima, Terahertz timedomain spectroscopy of solids: A review, J. Infrared Millim. Te. 26, 1661 (2005).

[43] J. C. Slonczewski, Conductance and exchange coupling of two ferromagnets separated by a tunneling barrier, Phys. Rev. B 39, 6995 (1989).

[44] C. Vicario, C. Ruchert, F. Ardana-Lamas, P. M. Derlet, B. Tudu, J. Luning, and C. P. Hauri, Off-resonant magnetization dynamics phase-locked to an intense phase-stable terahertz transient, Nat. Photonics 7, 720 (2013).

[45] G. Torosyan, S. Keller, L. Scheuer, R. Beigang, and E. Th. Papaioannou, Optimized spintronic terahertz emitters based on epitaxial grown Fe/Pt layer structures, Sci. Rep. 8, 1311 (2018).

[46] M. B. Jungfleisch, Q. Zhang, W. Zhang, J. E. Pearson, R. D. Schaller, H. Wen, and A. Hoffmann, Control of Terahertz Emission by Ultrafast Spin-Charge Current Conversion at Rashba Interfaces, Phys. Rev. Lett. 120, 207207 (2018).

[47] C. Zhou, Y. P. Liu, Z. Wang, S. J. Ma, M. W. Jia, R. Q. Wu, L. Zhou, W. Zhang, M. K. Liu, Y. Z. Wu, and J. Qi, Broadband Terahertz Generation via the Interface Inverse Rashba-Edelstein Effect, Phys. Rev. Lett. 121, 086801 (2018).

[48] J. G. Li, Z. M. Jin, B. J. Song, S. N. Zhang, C. Y. Guo, C. H. Wan, X. F. Han, Z. X. Cheng, C. Zhang, X. Lin, G. H. $\mathrm{Ma}$, and J. Q. Yao, Magnetic-field-free terahertz emission from a magnetic tunneling junction, Jpn. J. Appl. Phys. 58, 090913 (2019).

[49] Y. Ogasawara, Y. Sasaki, S. Iihama, A. Kamimaki, K. Z. Suzuki, and S. Mizukami, Laser-induced terahertz emission from layered synthetic magnets, Appl. Phys. Express 13, 063001 (2020). 